\title{
Serotonin regulation of behaviour via large-scale neuromodulation of serotonin receptor networks
}

Piergiorgio Salvan ( $\Delta$ piergiorgio.salvan@gmail.com )

University of Oxford https://orcid.org/0000-0003-3947-9541

\section{Madalena Fonseca}

University of Oxford https://orcid.org/0000-0002-5641-1306

Anderson Winkler

https://orcid.org/0000-0002-4169-9781

Antoine Beauchamp

Hospital for Sick Children

Jason Lerch

Wellcome Centre for Integrative Neuroimaging

Heidi Johansen-Berg

University of Oxford https://orcid.org/0000-0002-4134-9730

\section{Article}

Keywords:

Posted Date: January 7th, 2022

DOI: https://doi.org/10.21203/rs.3.rs-1019411/v1

License: (c) (i) This work is licensed under a Creative Commons Attribution 4.0 International License. Read Full License

Version of Record: A version of this preprint was published at Nature Neuroscience on December 15th, 2022. See the published version at https://doi.org/10.1038/s41593-022-01213-3. 


\section{Abstract}

Although we understand how serotonin receptors function at the single-cell level, what role different serotonin receptors play in regulating brain-wide activity and, in turn, human behaviour, remains unknown. Here, we developed transcriptomic-neuroimaging mapping to characterise brain-wide functional signatures associated with specific serotonin receptors: serotonin receptor networks (SRNs). Probing SRNs with optogenetics-fMRI and pharmacology in mice, we show that activation of dorsal raphe serotonin neurons differentially modulates the amplitude and functional connectivity of different SRNs, showing that receptors' spatial distributions can confer specificity not only at the local, but also at the brain-wide, network-level. In humans, using resting state fMRI, different sets of SRNs are linked to different behavioural phenotypes. These results provide compelling evidence that heterogeneous brainwide distributions of different serotonin receptor types may underpin behaviourally-distinct modes of serotonin regulation. This suggests that dorsal raphe serotonin neurons may regulate multiple aspects of human behaviour via modulation of large-scale receptor networks.

\section{Introduction}

Investigating the relationship between large-scale brain activity and behaviour can inform us on how a vast array of human behaviours arises from the coordinated activity between neural populations ${ }^{1-3}$. However, neurochemical modulation can bias neural activity by regulating neuronal excitability and plasticity and thus, in turn, affect behaviours ${ }^{4}$. Understanding how neuromodulation orchestrates brainwide activity is important as it may provide new insight into the regulation of multiple aspects of human behaviours in health and disease.

Serotonin regulates behaviour by modulating neuronal excitability and plasticity, and its dysfunction has been implicated in several neurologic and psychiatric diseases, such as anxiety and depression ${ }^{5-7}$. Serotonin is produced by a surprisingly small proportion of neurons (less than $0.1 \%$ of brain neurons) primarily located in the dorsal raphe nucleus (DRN), but it is released widely throughout the brain ${ }^{8}$. In the synaptic cleft, serotonin can interact with multiple receptor types that can vary in spatial distribution, chemical affinities, and cellular effects ${ }^{9-11}$. Despite being implicated in a dizzying array of phenomena, a comprehensive theory of how the serotonin system is functionally organised at the macroscopic brainwide level to support diverse functions remains elusive ${ }^{12}$. While the effects of different receptor types at the local-level are known, the importance of their brain-wide distribution patterns remains poorly understood. In particular, whether the different spatial patterns of serotonin receptor types provide a macroscale principle of organisation for the diverse regulation of human behaviour, remains unknown.

The DRN serotonin system has historically been treated as a monolithic population, yet recent evidence suggests that functional sub-systems may exist ${ }^{13}$. Recent accounts of the behavioural effects of activating DRN serotonin neurons have provided diverse and somewhat conflicting results, with implicated behaviours including reinforcement ${ }^{14}$, reward and punishment ${ }^{15}$, patience and delayed 
reward ${ }^{16-18}$, promotion of anxiety-like behaviour ${ }^{19,20}$, and suppression of locomotion ${ }^{21}$. This literature, together with growing evidence supporting the molecular heterogeneity of the DRN serotonin projection system 22-24, has suggested the existence of parallel serotonin sub-systems within the DRN. Recent work using viral-genetic methods has indeed provided strong evidence of the existence of anatomicallysegregated DRN serotonin projections, with similar responses to reward and opposite response to aversive stimuli ${ }^{13}$. However this dissection of the DRN serotonin system relies on the concept of anatomical presynaptic segregation, and does not consider the complex, heterogeneous nature of serotonin synapses, characterised by an intricate, complex tapestry of serotonin receptor types ${ }^{25,26}$.

A prominent view theorises that neuromodulation regulates different behavioural circuits via different receptor types, with distinct spatial distributions, and with different affinities that endow sensitivity to different timescales and input characteristics ${ }^{27}$. Whilst we understand how serotonin receptors function at the single-cell level ${ }^{25,26}$, we do not yet understand how these receptors affect brain-wide activity and, in turn, behaviour. This is fundamental to better understand serotonin's role both in health and disease.

To understand how different serotonin receptors shape serotonin regulation of brain-wide activity and affect behaviour, requires consideration of both spatio-temporal dynamics of serotonin neuromodulation and variation in human behaviour. Neuroimaging provides measurements that are sensitive to cellular phenomena and that can also be acquired in living humans, allowing us to bridge between cellular mechanisms investigated in animal models and human population variation in brain and behaviour. Here, we combined neuroimaging and gene expression maps to extract brain-wide functional signatures associated with specific serotonin receptors to investigate how different serotonin receptor types shape brain-wide activity and, ultimately, behaviour. First, by combining optogenetics, pharmacology, wholebrain imaging, and gene expression maps in mice, we test whether distinct brain-wide networks, characterised by different serotonin receptor types (serotonin receptor networks, SRNs), are differentially modulated by serotonin manipulations. Next, using the same neuroimaging phenotypes in humans, we ask whether variability in SRNs can account for population variation in human behaviour. This work provides a mechanistic understanding of how DRN serotonin actions on different serotonin receptor types may mediate the regulation of distinct phenotypes of human behaviours.

\section{Results}

\section{Mapping spatio-temporal fMRI signatures of brain-wide serotonin receptor networks (SRNs)}

In order to determine functional signatures associated with different serotonin receptors we developed a transcriptomic-neuroimaging approach that combined mouse and human gene expression brain maps from the Allen Institute with functional-MRI (fMRI) (Fig. 1). Using optogenetic-fMRI (ofMRI) data in mice and rs-fMRI data in humans, we characterised spatio-temporal fMRI signatures associated with specific serotonin receptors. We refer to these signatures as serotonin receptor networks (SRNs). 
We leveraged publicly available mouse and human brain transcriptomic maps of serotonin receptor genes $\left(\mathrm{Htr} 1-7 ;{ }^{28,29}\right)$. Each map characterises the brain-wide gene expression level for a single serotonin receptor gene. We used the established tool FSL Dual Regression (DR) ${ }^{30}$, to relate these gene expression maps to fMRI. For each subject, FSL DR first computes a SRN-specific time-course (reflecting the amplitude of network activity; DR-stage 1; Fig. 1a) then a functional connectivity spatial map (reflecting the spatially distributed nature of correlations; DR-stage 2; Fig. 1b). FSL DR-stage 1 and 2 thereby provide temporal and spatial fMRI signatures of different serotonin receptor genes, respectively. Greater correlation in fMRI time-courses in brain areas with similar gene expression level, will result in greater SRN amplitude changes and functional connectivity. At the group-level, human SRN functional connectivity maps showed a good spatial correspondence with group-level serotonin receptor density maps previously characterised via PET (Fig. S1) ${ }^{31}$. Importantly, this transcriptomic-neuroimaging mapping approach can be equally applied to mice ofMRI data and human rs-fMRI data, thus providing translatable neuroimaging phenotypes.

\section{Optogenetics activation of serotonin DRN neurons in mice elicits different amplitude changes in brain SRNs}

Given the different spatial distribution and response properties of different receptors, it is plausible that the same serotonin manipulation will differently modulate distinct SRNs. Here, in mice, we tested the hypothesis that optogenetics activation of DRN serotonin neurons elicits distinct changes in brain-wide SRNs. To test this hypothesis we used existing publicly available data from an ofMRI manipulation of ePet-Cre mice expressing channelrhodopsin-2 (ChR2) in DRN serotonin neurons (Fig. 2a) ${ }^{32}$, and reanalysed it with the novel transcriptomic-neuroimaging approach described above. Whilst ofMRI allows for precise causal manipulation of DRN serotonin neurons and concurrent recording of brain-wide function, transcriptomic-neuroimaging mapping of serotonin receptor genes (Htr1-5; Fig. 2b; ${ }^{28}$ ) allows to determine how different SRNs contribute to the brain-wide effect of activating DRN serotonin neurons. Using transcriptomic-ofMRI mapping, we can extract one time-series (Fig. 2c-e, Fig. S2a) and one brainwide functional connectivity map (Fig. 2f) for each receptor gene, for each experimental mouse.

First, we investigated whether $20 \mathrm{~Hz}$ optogenetic stimulation of DRN serotonin neurons causes amplitude fluctuations in brain-wide SRNs. Although the DRN system is known to co-release other neurotransmitters, DRN stimulation at $20 \mathrm{~Hz}$ is known to have predominant serotonergic effects rather than glutamatemediated ${ }^{33}$. Here, in ChR2 mice, we observed that activating DRN serotonin neurons gave rise to a variety of time-locked network amplitude changes (output from DR-stage 1) in SRNs (Fig. 2d). These network amplitude changes were not present in control mice. Using permutation-based inference testing (see Methods), we found that whilst Htr2c and Htr1a SRNs amplitude changes significantly increased due to optogenetic stimulation compared to controls, $\mathrm{Htr} 3 \mathrm{a}$ and $\mathrm{Htr} 3 \mathrm{~b}$ responses significantly decreased (statistical significance was assessed with 1,000 block-aware permutations, with family wise error rate correction (FWE-corr) across time, SRNs, and two tails; Fig. 2e). This dichotomy between the pair Htr1a$\mathrm{Htr} 2 \mathrm{c}$ and $\mathrm{Htr} 3 \mathrm{a}-\mathrm{Htr} 3 \mathrm{~b}$ is particularly interesting as $5-\mathrm{HT} 3$ receptors are the only ionotropic receptors 
among all 14 serotonin receptors. We also found that Htr1b SRN amplitude changed over time (initial significant decrease followed by a significant increase), that Htr5b SRN amplitude changes showed a delayed increase, and that the Htr4 showed an increase in amplitude. These results show a heterogeneity of SRNs temporal amplitude changes in response to activation of DRN serotonin neurons.

Then, we investigated the SRN spatial correlates (DR-stage 2) of DRN serotonin activation. Using permutation-based inference testing we found that activation of DRN serotonin neurons could either increase or decrease functional connectivity, either locally or globally (Fig. 2f), independently of whether a SRN temporal response increased or decreased in amplitude. For example, whilst a significant temporal decrease in the Htr3a SRN amplitude resulted in decreased functional connectivity across brain regions, a similar decrease in temporal amplitude in the Htr1b SRN, resulted in increased functional connectivity. These results show that different brain areas, depending on the admixture of serotonin receptors they express, can show radically different $\mathrm{fMRI}$ responses to DRN serotonin neuron activation. Whilst heterogeneous responses would be expected, given the different cellular effects of different receptors, our non-invasive approach allows us to demonstrate how such differences play out across time, and throughout the brain, providing a unique window into brain-wide receptor-specific dynamics. In addition, these results raise the possibility that the heterogeneous spatial distributions of serotonin receptor types may reflect not only a way for a single neurotransmitter to differently influence different local circuits, but also a more global mechanism to coordinate activity within defined large-scale networks. In other words, a macroscale principle of organisation of serotonin neuromodulation.

\section{Fluoxetine manipulation of brain-wide SRN responses to DRN ofMRI}

To better understand serotonin regulation of brain-wide SRNs and further validate our approach, we then asked how selectively manipulating serotonin availability in synaptic terminals modulates SRNs. We took advantage of fluoxetine, a selective serotonin reuptake inhibitor (SSRIs), which is known to increase synaptic serotonin availability and is used as a major therapeutic option for psychiatric disorders. Although the effects of fluoxetine on brain activity are not completely understood, previous research suggests that certain serotonin receptor types may be particularly important for its therapeutic effects. The acute administration of SSRI is known to indirectly activate $5 \mathrm{HT} 1 \mathrm{~A}$ and $5 \mathrm{HT} 1 \mathrm{~B}$ receptors and, in turn, to inhibit DRN serotonin cell firing and to decrease extracellular serotonin as measured via in-vivo microdialysis ${ }^{34-36}$. Furthermore, previous work has shown that $5 \mathrm{HT} 4$ receptor activation is necessary for the anxiolytic/antidepressant-like effects of fluoxetine ${ }^{26,37}$. Here, we leveraged our transcriptomicneuroimaging mapping approach described above to study the effect of fluoxetine on brain-wide SRNs. We made use of a publicly available dataset ${ }^{32}$, in which an acute, pharmacologically relevant dose (4.5 $\mathrm{mg} \mathrm{kg}-1^{38}$ ) of fluoxetine was administered via tail vein infusion during ofMRI (Fig. 3a). Using this dataset, we tested the hypothesis that fluoxetine significantly changed DRN modulation of the Htr1a, $\mathrm{Htr} 1 \mathrm{~b}$, and Htr4 SRNs. Using permutation-based inference testing we found a bidirectional effect of fluoxetine: whilst fluoxetine downregulated DRN modulation of Htr1a and Htr1b SRNs' amplitude response, it upregulated DRN modulation of the Htr4 SRN (Fig. 3b, c; although these effects did not significantly alter SRNs' functional connectivity: all p-values $>0.05$ ). We also found that the same 
fluoxetine manipulation of DRN modulation of the Htr1a SRN did not significantly differ from the control group (Fig. S3a). Of interest, we highlight a negative correlation between Htr1a and Htr4 SRNs, both across time and across subjects (Fig. S3b).

These results are supported by previous studies showing that 5-HT1A receptor antagonists prevent the acute inhibitory effect of SSRI on DRN cell firing, thus augmenting the effect of fluoxetine ${ }^{39,40}$. These results are also aligned with previous literature showing that serotonin 5-HT4 receptor mediated synaptic potentiation plays a central role in fluoxetine's antidepressant actions ${ }^{41,42}$. Furthermore, short-term treatment via 5-HT4 receptor agonists mimics the cellular, molecular, and behavioural antidepressant effects achieved after chronic SSRI administration ${ }^{37,43}$, and further potentiates SSRIs effects ${ }^{44}$. Crucially, 5-HT4 receptor antagonists are known to block the anxiolytic effect of fluoxetine ${ }^{37}$, demonstrating a key role for this pathway. These results show that combining transcriptomic maps with pharmacological ofMRI, can capture spatio-temporal effects known to exist in the current literature, and therefore provide a strong validation of the mapping approach described here to study serotonin neuromodulation and the role of different receptor types.

\section{Human behaviours and brain SRNs are functionally organised into independent modes of population variation}

Serotonin has been implicated in a myriad of cognitive and behavioural functions, yet a comprehensive understanding of how this is achieved at the brain-wide level is still lacking. The existence of multiple serotonin receptors with different cellular effects offers a mechanism by which a single neuromodulator can have diverse downstream effects. Yet, how this palette of receptors is used at the whole-brain level to regulate different behaviours remains a mystery.

Building on the transcriptomic-neuroimaging mapping approach developed above, here we studied the link between human brain functional organisation of SRNs and human behaviour at the population level. Using data from the Human Connectome Project ${ }^{45}$, we tested whether differences in SRNs functional connectivity could account for individual differences in specific sets of behavioural and cognitive functions, such as emotional processing, delay discounting, and others.

To do this, we combined human brain transcriptomic maps of serotonin receptor genes (HTR1-7) with resting state BOLD fMRI data from 812 subjects $^{2}$ using the FSL DR approach described above (DR-stage 2) (Fig. 4a). This allowed us to quantify subject-specific functional connectivity strength for each SRN, thus characterising individual differences in the brain-wide functional organisation of SRNs. 333 variables describing cognition, behaviour, familiar history, etc., from 30 different categories of human behaviour (as previously used in ${ }^{46}$ ), were then considered for SRNs-behaviour covariation analysis (Fig. 4b).

Using canonical correlation analysis (CCA), we investigated modes of population covariation between SRNs and behaviour, and assessed their statistical significance using permutation methods ${ }^{47}$. We found 
three strong modes of population variation linking distinct sets of SRNs with distinct sets of human behaviour (Fig. 4c-f; CCA mode 1, $r=0.77$; FWE-corr $p$-value = 0.001; CCA mode 2, $r=0.75$; FWE-corr $p$ value $=0.004 ;$ CCA mode $3, r=0.74 ; F W E$-corr $p$-value $=0.019$; statistical significance assessed via 1,000 block-aware permutations). These three orthogonal modes characterise a relationship between human behaviour and SRNs functional connectivity as organised into three independent modes of individual variation (Fig. 4f-g). Each mode is thus characterised by distinct pairs of behavioural (Fig. $\mathbf{4 f - g}$ ) and neuroimaging phenotypes (Fig. $\mathbf{3 4 g}$, Fig. S4a). Of interest, we highlight that the first mode of covariation showed strong cross-loadings in behavioural variables related to delay discounting (Fig. 4d-e), and by greater positive loadings in the SRNs HTR1E, HTR1F, and HTR2A, underscoring the role that different serotonin receptor types may play in mediating the effect of serotonin on patience and reward processing. The second mode was characterised by greater loadings in literacy, fluid intelligence, and emotional processing, paired with a prefrontal pattern of SRN functional connectivity. The third mode was characterised by involvement in domains including semantics, social skills, language, and working memory, coupled with strong positive loading for HTR5A, and a pattern of cerebellar and subcortical functional connectivity.

Together these findings show that serotonin has diverse and wide reaching associations with human behaviour, and that its effects are organised into multiple, independent modes of brain-behaviour modulation. These results suggest that at the human population level, the regulation of different behavioural domains may be achieved via serotonin modulation of different serotonin receptor types. Hence these results provide an opportunity for hypothesis generation on the multiple links between serotonin receptors and human behaviour and cognitive functions.

\section{Discussion}

In this work, we study the large-scale organisation of serotonin neuromodulation and the role of different serotonin receptor types in mediating serotonin's effects on brain-wide activity and behaviour. We do this across mice and humans, by using a transcriptomic-neuroimaging approach that allows us to characterise brain-wide fMRI signatures of serotonin receptor genes that are known to have heterogeneous spatial expression patterns. We call these fMRI signatures SRNs. In mice, we show that activation of DRN serotonin neurons elicits heterogeneous responses, in both response amplitude and functional connectivity, across different SRNs. We then show how fluoxetine has bidirectional effects on the DRN serotonin modulation of specific SRNs, as would be predicted from previous pharmacological studies. In humans, we then ask whether individual differences in the large-scale functional organisation of these SRNs underpin population variation in human behaviour. Using population brain imaging from the HCP dataset, we show that individual differences in SRNs functional connectivity are organised in three independent modes of population variation, each coupled with different domains of human behaviour. This suggests that different behavioural domains may undergo different serotonin regulation depending on the pattern of serotonin receptor types expressed in the relevant circuits. 
Our findings suggest that serotonin brain-wide neuromodulation is a far more complex and multifaceted phenomena than previously thought ${ }^{32,48}$. Driving DRN serotonin neuromodulation through means of ofMRI, allowed us to study how different SRNs differ in their responses (network amplitude and functional connectivity changes). Different serotonin receptors are expressed with partially overlapping distribution densities in the brain, have different cellular effects, and benefit from different affinities to serotonin concentrations ${ }^{27}$. These dissimilarities may explain why we observe a heterogeneity in SRN responses and, in turn, may suggest why different brain areas - characterised by different admixtures of receptor types - may have different sensitivities to serotonin neuromodulation. Indeed one further possibility is that heterogeneous, brain-wide distribution densities of different serotonin receptor types represent a large-scale principle of organisation for serotonin regulation of brain networks and behaviours. Crucially, we tested this hypothesis here via population level brain imaging in humans. We found that domains of human behaviour, like decision making or emotional processing, are differentially associated with different sets of SRNs functional connectivity across individuals. Indeed previous work has hinted at the existence of an heterogeneous link between serotonin receptor types and behaviour. Studies in mice have implicated polymorphism in different serotonin receptor genes with a diverse modulation of behavioural function ${ }^{49}$. Furthermore, genetic knockout models of different serotonin receptor genes express contrasting behavioural phenotypes when examined on a number of behavioural paradigms ${ }^{50}$. Serotonin synapses are present brain-wide, and are characterised by a complex tapestry of different receptor types that are encoded by 7 gene families ${ }^{5}$. As serotonin neuromodulation is systemic, and dramatic differences in patients' responses to SSRI exist ${ }^{5,12}$, our findings suggest that interindividual variation in serotonin regulation may be mediated by differences in the brain-wide functional organisation at the serotonin receptor level.

Of interest, our findings highlight the link between SRN dynamics and human individual variation in delay discounting, the tendency to choose smaller but immediate rewards over larger but delayed ones. Although the precise behavioural functions of serotonin are not yet well understood, serotonin is well known to play a key role in patience and delayed reward ${ }^{16-18}$. Here we found that greater functional connectivity in the SRN HTR2A (as well as HTR1E and HTR1F; CCA mode 1) was linked to smaller delay discount across individuals. Indeed serotonin has been implicated in this decision making tendency, with lower serotonin levels linked to greater temporal discounting in humans ${ }^{51}$. The idea that serotonin effects on delay discounting may depend on the regulation of the serotonin 5 HT2A receptor pathway is consistent with previous literature showing its involvement in delay discounting in humans ${ }^{52}$. Furthermore, in mice, a link between 5HT2A receptor levels and individual variation in impulsivity traits has been shown to exist ${ }^{53}$. Our findings provide further support for the notion that the brain-wide functional organisation of serotonin receptors may mediate serotonin's regulation of intertemporal choices. This suggests that polymorphisms in serotonin receptors may influence human biases in decision making by altering serotonin interactions with different receptors.

In this work we exploited the power of cross-species neuroimaging in order to bridge mechanistic insight in mice with population brain imaging in humans, and unveiled the large-scale functional organisation of 
serotonin neuromodulation. Whilst fMRI is best suited for recording mixed signals of brain-wide activity, transcriptomic maps of serotonin receptor genes (both for the human and the mouse brain) provide the parameters to decode the signals arising from the intricate and diffuse spatial patterns of serotonin receptor types. OfMRI then provides the perfect tool to precisely activate DRN serotonin neurons and, together with SRN maps, allows us to implicate specific patterns of brain-wide SRN dynamics otherwise anatomically tangled - in DRN neuromodulation. These fMRI signatures are most likely the result of complex receptor signalling transduction pathways and thus caution is required in the interpretation of the underlying cellular events ${ }^{5}$. Indeed seven classes of serotonin receptors exist, most of which are G protein-coupled receptors (GPCR) (with the exception of 5-HT3 receptors that are ionic channels) and the complexity of their effects scales by the number of proteins they interact with 25,26 . These receptors are the target of many therapeutic drugs for treating several psychiatric disorders ${ }^{5}$. Given the widespread prevalence of such conditions worldwide, further efforts are needed to better understand how SRNs play a role in mediating brain-wide neuromodulation, both in health and disease.

Although the idea of enriching fMRI with molecular information is not new in human neuroimaging ${ }^{54-57}$, here we used transcriptomic-neuroimaging mapping to study the effects of an acute pharmacological manipulation on DRN serotonin optogenetic activation. We showed that the SSRI fluoxetine evoked bidirectional changes in the effect of optogenetic manipulation on the SRNs. Firstly, we found that fluoxetine downregulated the optogenetic effect on Htr1a and Htr1b SRNs. Crucially, this finding is predicted by previous literature implicating the hyperpolarization of serotonin autoreceptors as a key effect of acute SSRIs administration ${ }^{36}$. Indeed whilst acute administration of fluoxetine inhibits firing of DRN serotonin neurons, chronic administration (>7 days) gradually recovers DRN firing ${ }^{58}$. This is known to occur due to desensitization of autoreceptors with repeated SSRI exposure ${ }^{36}$. It will be interesting to test whether the downregulation of the optogenetic effect on Htr1a SRNs seen with acute SSRI changes with chronic exposure, and over which timescales. Secondly, we found that fluoxetine upregulated DRN activation of Htr4 SRN. Importantly, the 5HT4 receptor pathway has previously been shown to play a necessary causal role in the action of fluoxetine ${ }^{37}$. Furthermore, over long timescales (weeks), central serotonin levels are inversely related to $5 \mathrm{HT} 4$ receptor density. Chronic administration of fluoxetine is indeed known to downregulate $5 \mathrm{HT} 4$ receptor density, both in rodents ${ }^{59}$ and humans ${ }^{60}$, via desensitization of 5HT4 receptor-dependent signalling. The results of this work therefore also complement previous literature on $5 \mathrm{HT}$ receptors ${ }^{26}$ and suggest the possibility that the inverse relation between central serotonin levels and 5-HT4 receptors may exist even over short timescales (hours). Indeed it has previously been hypothesised that 5-HT4 receptors interact with presynaptic serotonin autoreceptors in a negative feedback control loop ${ }^{36}$. Together, these results show that combining transcriptomic-fMRI mapping with pharmacological ofMRI may provide novel neuroimaging markers for serotonin synaptic effects, opening exciting new avenues for personalised medicine.

Our mapping approach between gene expression maps and fMRI has some limitations. Firstly, it is correlational and relies on the fact that different serotonin receptors have partially overlapping distribution densities. Therefore it is limited by the reliability of characterising brain-wide gene expression 
levels ${ }^{28,29}$. The approach used here builds upon previous, established approaches using gene expression maps together with structural MRI to study the biological pathways underlying brain network organisation ${ }^{61,62}$. Here we use the same state-of-the-art brain-wide gene expression maps ${ }^{28,29}$ to study the brain network functional organisation. Secondly, our approach is limited by using transcriptomic atlases to infer receptor distributions. PET would arguably be better suited to capture individual differences in receptor density. Yet this approach would not allow access to either the spatio-temporal dynamics during neuromodulation, or the sample sizes needed to investigate variation at the population level - as previously highlighted ${ }^{54}$. Future effort should therefore be tailored to overcome these limitations by studying how invasive manipulation of specific receptor pathways (e.g. blockage) alters networks of serotonin receptors at the brain-wide level and how individual variation in receptor densities may play a role. Thirdly, dorsal raphe neurons are not the only source of brain serotonin. The serotonin median raphe neurons are also known to play an important role in serotonin brain regulation by virtue of their complementary projection pattern 24,63 . This highlights the need to characterise similarities and differences between these two nuclei, their interactions with different serotonin receptor types, and their effects on different behaviours.

By reinforcing the importance of cross-species translational research, our results bridge the gap between serotonin manipulations of brain-wide dynamics and population variation in behaviour. The findings of this work show that heterogeneous brain-wide patterns of different serotonin receptor types represent a macroscale principle of organisation for serotonin regulation of brain networks and behaviours. Whether other neuromodulatory systems share similar organisational principles remains unknown. Furthermore, whether genetic polymorphisms endow individual variation in SRNs, thus mediating a propensity to developmental and psychiatric disorders or responsiveness to drug treatments, remains unclear. Answering these questions via population medical imaging and genetics may offer novel chances for personalised medicine and drug discovery.

\section{Declarations}

\section{Acknowledgements}

We thank Professors Trevor Sharp, David Bannerman, and Mark Walton, for many useful comments on the manuscript. We thank the members of the WU-Minn-Ox Human Connectome Project Consortium for freely sharing the data with the scientific community. We are also grateful to the Authors of Grandjean et al. (2019) for supporting open-science initiatives by making their data publicly available. H.J.B. is supported by a Wellcome Principal Research Fellowship (110027/Z/15/Z). J.L. and M.F. are supported by the Wellcome Centre for Integrative Neuroimaging. The Wellcome Centre for Integrative Neuroimaging is supported by core funding from the Wellcome Trust (203139/Z/16/Z). This research was funded in whole, or in part, by the Wellcome Trust (110027/Z/15/Z). For the purpose of Open Access, the author has applied a CC BY public copyright licence to any Author Accepted Manuscript version arising from this submission. 


\section{Author Contributions}

Conceptualization, P.S., J.L. and H.J.B.; Software, A.M.W.; Formal Analysis, P.S.; Writing - Original Draft, P.S., J.L. and H.J.B.; Writing - Review \& Editing, P.S., M.F., A.M.W., J.L. and H.J.B.; Funding Acquisition, J.L. and H.J.B.; Resources, A.B.; Supervision, J.L., and H.J.B.

\section{Declaration of Interests}

The authors declare no competing financial interests.

\section{Methods}

\section{Mouse neuroimaging}

\section{Concurrent optogenetic-fMRI (ofMRI) of DRN serotonin neurons in mice}

We used concurrent Cerebral Blood Volume (CBV) ofMRI data from previously published in Grandjean et al. ${ }^{32}$. This data is publicly available and can be found at OpenNeuro (Project_ID: Mouse_opto_DRN; raw ofMRI data). We used data from the experiments with ofMRI manipulation of ePet-Cre mice expressing channelrhodopsin-2 (ChR2) in DRN serotonin neurons $(N=8$, runs $=63)$, controls expressing eYFP only ( $N$ $=4$, runs $=18)$, and those ePet-Cre mice treated with fluoxetine prior to ofMRI $(\mathrm{N}=6$, runs $=18)$. MRI acquisition, data preprocessing, and all surgical procedures are described in detail in Grandjean et al. ${ }^{32}$. Briefly, the light-sensitive ion channel channelrhodopsin-2 (ChR2) was expressed in DRN Pet-1 serotonin neurons using a cre-dependent adeno-associated virus (AAV-EF1a-DIO-ChR2-EYFP) injected into the DRN of ePet-cre+/- mice. Light was delivered through an MRI-compatible optical fibre, implanted in the DRN $(\mathrm{ML}=0, \mathrm{AP}=-0.6 \mathrm{~mm}$ from Lambda, $\mathrm{DV}=3.3 \mathrm{~mm}$ from the skull), 1-2 weeks post-viral infection and at least 1 week prior to ofMRI. As controls, ePet-cre+/- mice underwent the same procedures except that they received a virus lacking ChR2 (AAV-EF1A-DIO-EYFP). Functional MRI was performed in a 7T Bruker scanner equipped with a surface coil in mice anaesthetised with a mixture of isoflurane $(0.5 \%)$ and medetomidine $(0.1 \mathrm{mg} / \mathrm{kg}$ bolus followed by a constant infusion of $0.2 \mathrm{mg} / \mathrm{kg} / \mathrm{h}$ s.c.). Mice were also injected with a paramagnetic iron oxide nanoparticle-based intravascular contrast agent (Endorem, $30 \mathrm{mg} / \mathrm{kg} \mathrm{Fe}$ ). Each fMRI run consisted of 6 cycles of 20 s of blue light stimulation at $20 \mathrm{~Hz}$ (pulse width $=$ $5 \mathrm{~ms}$, laser power $=40 \mathrm{~mW} / \mathrm{mm}^{\wedge} 2$ ) followed by 40 s of rest. Functional images were acquired with a spatial resolution of $0.31 \times 0.27 \times 0.5 \mathrm{~mm}$ and a temporal resolution of 2 seconds using a multi-shot gradient echo EPI sequence. Data preprocessing was performed using FSL and AFNI. Anatomical images from each scan session were used to generate a reference template. Linear and non-linear transformations were then estimated between the anatomical images and the reference template. Functional images were temporally realigned, transformed to match the reference template, and smoothed using a $0.45 \mathrm{~mm}^{\wedge} 2$ kernel. Time-series were summarised using the Allen Brain Atlas as in Grandjean et al. ${ }^{32}$ and sign inverted. The Allen Institute for Brain Science (AIBS) mouse brain atlas was resampled to 90 regions-of-interest by merging leafs (e.g., cortical layers) by branches (e.g., cortical area). 
The nomenclature, and abbreviations for the brain regions are in accordance with https://atlas.brainmap.org/.

\section{Transcriptomic-neuroimaging mapping in mice}

We used mouse brain-wide gene expression maps of serotonin receptor genes publicly available from the Allen Brain Institute ${ }^{28,29}$. Gene maps were summarised using the Allen Mouse Brain Atlas as in Grandjean et al. ${ }^{32}$. Most transcriptomic maps of serotonin receptor genes were available in coronal sections with expression levels across the whole brain (Htr1a, Htr1b, Htr2c, Htr3a, Htr3b, Htr5b). Those that were available in sagittal sections with expression levels for one single hemisphere (Htr1f, Htr2a, $\mathrm{Htr} 4$ ) were thus flipped along the $X$ axis and made symmetric. Each serotonin receptor gene map (Htr1-5), representing the raw expression level for each gene across the whole-brain, was then log2-transformed and Z-scored across brain regions. Then we used FSL Dual Regression (DR; a multiple linear regression method; https://fsl.fmrib.ox.ac.uk/fsl/fslwiki/DualRegression) ${ }^{30}$ in order to combine serotonin receptor gene maps together with individual ofMRI data. We standardised the stage 1 time-courses thus allowing us to overcome the network amplitude (or scale) problem ${ }^{30}$ and allowing us to compare network amplitude changes across subjects and conditions. This transcriptomic-neuroimaging approach allows to characterise 1) a time-course representing the network amplitude changes in fMRI activity (DR-stage 1), and 2) a functional connectivity map (DR-stage 2), for each animal, for each serotonin receptor gene. We refer to these signatures as Serotonin Receptor Networks (SRNs). A graphical representation of this approach can be found in Fig. 1.

In order to correct SRNs amplitude changes to optogenetic stimulation for baseline differences, we subtracted the average SRNs activation during the 40 ofMRI volumes prior to the first stimulation block from the remaining SRN amplitude time-courses (see Fig. S2a). We opted for this baseline instead of all the "resting" periods during the stimulation blocks as the elicited SRNs amplitude changes are longlasting way beyond the stimulation phase.

\section{Permutation inference testing via general linear models (GLMs)}

All inference testing on ofMRI DR-stage 1 and stage-2 outputs (network amplitude changes and functional connectivity maps) was carried out using FSL Permutation Analysis of Linear Models (PALM v119: PALM - FsIWiki ${ }^{64}$ ). The null distribution was characterised with 1,000 permutations. Statistical significance was established based on family wise error rate (FWE)-corrected p-values. When testing inferences on SRN temporal responses to optogenetic stimulation (DR-stage 1) (i.e. group comparisons: ChR2 vs control animals), one-dimensional (time) threshold-free cluster enhancement (TFCE) was applied 65. P-values were FWE-corrected across time, network tested (SRNs), and two-tails inference ("greater/smaller amplitude change than"). When testing inferences on brain regions functional connectivity changes to optogenetic stimulation (DR-stage 2), no cluster enhancement was applied. Pvalues were FWE-corrected across atlas ROIs, network tested (SRNs), and two-tails inference ("greater/smaller functional connectivity than"). When testing the effect of fluoxetine, because of the 
within subject design, we constrained the permutations to be block-aware, thus allowing permutations only within the same subject across conditions ${ }^{66}$. All statistical significance results are plotted as $-\log _{10}$ of FWE-corr $\mathrm{P}$ and results were deemed significant at FWE-corr $\mathrm{P}<0.05$. All statistical analyses were carried out in MATLAB 2020. The results of these analyses are shown in Fig. 2, 3, S2, and S3.

\section{Human neuroimaging}

\section{Resting state (rs)-fMRI in the Human Connectome Project (HCP)}

We used resting state BOLD (Blood Oxygen Level Dependent) fMRI data from $N=812$ subjects from the $\mathrm{HCP}$, which provides the required ethics and consent needed for study and dissemination, such that no further additional institutional review board (IRB) approval is required. These are all subjects with complete resting fMRI data, all healthy adults (aged 22-35 years, 410 females) scanned on a 3-T Siemens connectome-Skyra. For each subject, four 15-min runs of fMRI time series data with a temporal resolution of $0.73 \mathrm{~s}$ and a spatial resolution of 2-mm isotropic were available. The preprocessing pipeline followed the technique of Smith et al. ${ }^{67,68}$, and thus will be described only briefly here. Spatial preprocessing was applied using the procedure described by Glasser et al. ${ }^{69}$. We applied structured artefact removal using ICA followed by FMRIB's ICA-based X-noisefier (FIX) from the FMRIB Software Library (FSL) ${ }^{70}$, which removed more than $99 \%$ of the artefactual ICA components in the dataset. We did not use global signal regression. This resulted in 812 subjects, each having 4 rs-fMRI of 1,200 time points.

\section{Transcriptomic-neuroimaging mapping in humans}

We used serotonin receptor gene brain maps from the Allen Human Brain Atlas (AHBA) $\left(\mathrm{Htr} 1-7 ;{ }^{29}\right)$. The microarray data sets were processed as described in Arnatkevičiūtė, Fulcher and Fornito (2019) ${ }^{71}$. Specifically, microarray probes from each of the six donors in the AHBA were initially filtered to retain probes with existing entrez gene IDs. The remaining probes were subsequently filtered using the AHBA intensity-based filtering binary indicators, such that the probes for which fewer than $50 \%$ of the samples passed the filter were discarded. For every donor, the expression values of multiple probes were then averaged when those probes corresponded to the same gene. These averages were computed in linear space, and the aggregated values were subsequently transformed back to log space using a log2 transformation. The resulting gene-by-sample expression matrices were annotated such that the samples were mapped to the structure labels of an atlas parcellation. The atlas labels were assigned to samples on the basis of minimal Euclidean distance in MNI coordinate space. To do so, we used a novel atlas containing 152 cortical and subcortical regions, which was generated by merging the AAL cortical atlas ${ }^{72}$ with the 5 -atlas subcortical ${ }^{73}$, cerebellum ${ }^{74}$, colin27 thalamus and striatum ${ }^{75}$, hippocampus subfields ${ }^{76}$, and amygdala ${ }^{77}$ atlases from CoBrALab. The expression of every gene was then averaged over multiple samples with common atlas labels. This was done in linear space before converting back to $\log$ space with a log2 transformation. Finally, the structure-wise gene expression values were averaged 
across the two donors in the AHBA that have bilateral sampling (H0351.2001, H0351.2002), resulting in the final gene-by-region expression matrix.

For each of the 812 subjects, separately for each of the 4 rs-fMRI runs, FSL DR (with variance normalisation) was then used to combine brain maps of serotonin receptor genes (HTR1A, HTR1E, HTR1F, HTR2A, HTR2C, HTR3B, HTR4, HTR5A, HTR7) with rs-fMRI data at the voxel-wise level. (Serotonin receptor genes for the human brain are indicated in capital letters to distinguish from those of the mouse brain). SRN functional connectivity maps were estimated separately for each rs-fMRI dataset and then averaged across the 4 runs for each subject, resulting in a single functional connectivity map per SRN per subject.

Although SRN functional connectivity maps are at the voxel-wise level (see Fig. 4a), in order to ease the subsequent statistical analysis, for each SRN we summarised functional connectivity values based on the modified AAL atlas described above. This resulted in a subject $x$ atlas ROls $x$ SRNs matrix (812 subjects $\times 152$ ROIs $\times 9$ SRNs) that we fed to the following statistical analysis.

\section{Behavioural measures in the Human Connectome Project (HCP)}

We used the restricted behavioural data as provided by the HCP consortium in the CCA. We selected a list of 333 behavioural variables previously described by Llera et al. ${ }^{46}$. These variables represent a summary of demographic measures present in the HCP sample, and belong to 30 categories that we use to ease the interpretation of the findings (full detailed description can be found in van Essen et al. ${ }^{78}$ ).

\section{Permutation inference testing via canonical correlation analysis (CCA)}

In order to avoid an overdetermined, rank-deficient CCA solution, and to limit the chances of overfitting, a dimensionality reduction step was performed to both brain imaging and behavioural variables. Using the same approach previously applied by Salvan et al. ${ }^{79}$, brain imaging and behavioural variables were separately reduced using principal component analysis (PCA). Brain imaging variables were reduced into 116 PCs (variance explained > 95\%), whereas behavioural variables were reduced into 152 PCs (variance explained $>95 \%)$.

In order to study whether multiple modes of brain-behaviour covariation exist, we used CCA as implemented in Winkler et al. ${ }^{47}$ (https://github.com/andersonwinkler/PermCCA). This allowed us to test whether sets of SRNs were significantly related to behavioural phenotypes. Canonical correlations were estimated in a stepwise manner, removing at each iteration the variance already explained by previous modes of population covariation, while dealing with different numbers of variables in both sides. Crucially, this implementation of CCA also performs residualisation of confounds of no interest without introducing dependencies among the observations, which would violate the exchangeability assumption 47. Here, imaging and behavioural variables were adjusted for 17 confounding variables as previously performed by Smith et al., ${ }^{2}$ (1.acquisition reconstruction software version, 2.average subject head motion during rs-fMRI, 3.weight, 4.height, 5.systolic and 6.diastolic blood pressure, 7.hemoglobin A1C, 8.cube- 
root of total brain volume, 9.cube-root of total intracranial volume, as well as the squared version of measures 2-9 (the first is binary)). Statistical significance was tested with 1,000 block-aware

permutations respecting HCP family-structure ${ }^{66}$ and a family wise error correction was applied across all CCA modes. CCA imaging and behavioural cross-loadings were then extracted for all CCA modes deemed significant at FWE-corr $\mathrm{P}<0.05$. The results of these analyses are shown in Fig. 4.

To ease the interpretation of the results, the behavioural cross-loadings from 333 variables (Fig. 4c) were first Fisher-transformed (r-to-Z) (separately for each CCA covariate), converted to absolute values (for purposes of improving visualisation via radial plots), and averaged according to the 30 behavioural categories previously used by Llera et al. ${ }^{46}$. This allowed us to represent CCA behavioural phenotypes as behavioural fingerprints using a radial plot (Fig. 4 d).

CCA imaging cross-loadings were calculated for all brain regions and all SRNs (see Fig. S4) - these were not estimated at the voxel-wise level in order to preserve the spatial resolution used for statistical analysis. In order to ease interpretation, separately for each CCA mode, these maps were then 1) averaged across brain areas to give cross-loadings at the level of SRNs (plot displayed in Fig. 4f); and then 2) averaged across brain regions to give cross-loadings at the level of brain maps (maps displayed in Fig. $\mathbf{4 g}$ ). Both panels $\mathbf{f}$ and $\mathbf{g}$ in Fig. $\mathbf{4}$ display Z-scores to ease interpretation; instead Fig. $\mathbf{S 4}$ displays raw CCA imaging cross-loadings.

\section{Code availability}

- Most of the software and code used in this study are publicly available. Brain image processing and statistical testing was largely carried out with FSL (FMRIB's Software Library, https://fsl.fmrib.ox.ac.uk/fsl/fslwiki) and MATLAB-based code from FSLNets (https://fsl.fmrib.ox.ac.uk/fsl/fslwiki/FSLNets) and PermCCA (https://github.com/andersonwinkler/PermCCA).

- All original code will be deposited at GitHub and will be made publicly available as of the date of publication.

\section{Data availability}

Mouse ofMRI raw data are publicly available (raw fMRI data: https://openneuro.org/datasets/ds001541/versions/1.1.3; preprossed timeseries: http://dx.doi.org/10.34973/raa0-5z29). Human fMRI and behavioural data are publicly available at https://db.humanconnectome.org/. Transcriptomic data for both the mouse and human brain are publicly available at https://portal.brain-map.org/.

\section{References}


1. Finn, E. S. et al. Functional connectome fingerprinting: identifying individuals using patterns of brain connectivity. Nature Neuroscience vol. 18 1664-1671 (2015).

2. Smith, S. M. et al. A positive-negative mode of population covariation links brain connectivity, demographics and behavior. Nat. Neurosci. 18, 1565-1567 (2015).

3. Shine, J. M. et al. Human cognition involves the dynamic integration of neural activity and neuromodulatory systems. Nat. Neurosci. 22, 289-296 (2019).

4. Robbins, T. W. \& Arnsten, A. F. T. The neuropsychopharmacology of fronto-executive function: monoaminergic modulation. Annu. Rev. Neurosci. 32, 267-287 (2009).

5. Muller, C. P. \& Jacobs, B. Handbook of the Behavioral Neurobiology of Serotonin. (Academic Press, 2009).

6. Deneris, E. S. \& Wyler, S. C. Serotonergic transcriptional networks and potential importance to mental health. Nat. Neurosci. 15, 519-527 (2012).

7. Dayan, P. \& Huys, Q. Serotonin's many meanings elude simple theories. eL ife vol. 4 (2015).

8. Okaty, B. W., Commons, K. G. \& Dymecki, S. M. Embracing diversity in the 5-HT neuronal system. Nat. Rev. Neurosci. 20, 397-424 (2019).

9. Barnes, N. M. \& Sharp, T. A review of central 5-HT receptors and their function. Neuropharmacology vol. 38 1083-1152 (1999).

10. Hoyer, D., Hannon, J. P. \& Martin, G. R. Molecular, pharmacological and functional diversity of 5-HT receptors. Pharmacol. Biochem. Behav. 71, 533-554 (2002).

11. Mengod, G., Cortés, R., Teresa Vilaró, M. \& Hoyer, D. Distribution of 5-HT Receptors in the Central Nervous System. Handbook of Behavioral Neuroscience 123-138 (2010) doi:10.1016/s15697339(10)70074-6.

12. Cools, R., Roberts, A. C. \& Robbins, T. W. Serotoninergic regulation of emotional and behavioural control processes. Trends Cogn. Sci. 12, 31-40 (2008).

13. Ren, J. et al. Anatomically Defined and Functionally Distinct Dorsal Raphe Serotonin Sub-systems. Cell 175, 472-487.e20 (2018).

14. Liu, Z. et al. Dorsal Raphe Neurons Signal Reward through 5-HT and Glutamate. Neuron vol. 81 13601374 (2014).

15. Cohen, J. Y., Amoroso, M. W. \& Uchida, N. Serotonergic neurons signal reward and punishment on multiple timescales. Elife 4, (2015). 
16. Fonseca, M. S., Murakami, M. \& Mainen, Z. F. Activation of dorsal raphe serotonergic neurons promotes waiting but is not reinforcing. Curr. Biol. 25, 306-315 (2015).

17. Miyazaki, K. W., Miyazaki, K. \& Doya, K. Activation of Dorsal Raphe Serotonin Neurons Is Necessary for Waiting for Delayed Rewards. Journal of Neuroscience vol. 32 10451-10457 (2012).

18. Xu, S., Das, G., Hueske, E. \& Tonegawa, S. Dorsal Raphe Serotonergic Neurons Control Intertemporal Choice under Trade-off. Curr. Biol. 27, 3111-3119.e3 (2017).

19. Teissier, A. et al. Activity of Raphé Serotonergic Neurons Controls Emotional Behaviors. Cell Reports vol. 13 1965-1976 (2015).

20. Urban, D. J. et al. Elucidation of The Behavioral Program and Neuronal Network Encoded by Dorsal Raphe Serotonergic Neurons. Neuropsychopharmacology vol. 41 1404-1415 (2016).

21. Correia, P. A. et al. Transient inhibition and long-term facilitation of locomotion by phasic optogenetic activation of serotonin neurons. eLife vol. 6 (2017).

22. Okaty, B. W. et al. Multi-Scale Molecular Deconstruction of the Serotonin Neuron System. Neuron 88, 774-791 (2015).

23. Huang, K. W. et al. Molecular and anatomical organization of the dorsal raphe nucleus. Elife 8, (2019).

24. Ren, J. et al. Single-cell transcriptomes and whole-brain projections of serotonin neurons in the mouse dorsal and median raphe nuclei. Elife 8, (2019).

25. Lesch, K.-P. \& Waider, J. Serotonin in the Modulation of Neural Plasticity and Networks: Implications for Neurodevelopmental Disorders. Neuron vol. 76 175-191 (2012).

26. Sharp, T. \& Barnes, N. M. Central 5-HT receptors and their function; present and future. Neuropharmacology 177, 108155 (2020).

27. Dayan, P. Twenty-Five Lessons from Computational Neuromodulation. Neuron vol. 76 240-256 (2012).

28. Lein, E. S. et al. Genome-wide atlas of gene expression in the adult mouse brain. Nature $445,168-176$ (2007).

29. Hawrylycz, M. J. et al. An anatomically comprehensive atlas of the adult human brain transcriptome. Nature 489, 391-399 (2012).

30. Nickerson, L. D., Smith, S. M., Öngür, D. \& Beckmann, C. F. Using Dual Regression to Investigate Network Shape and Amplitude in Functional Connectivity Analyses. Front. Neurosci. 11, 115 (2017). 
31. Beliveau, V. et al. A High-Resolution In Vivo Atlas of the Human Brain's Serotonin System. The Journal of Neuroscience vol. 37 120-128 (2017).

32. Grandjean, J. et al. A brain-wide functional map of the serotonergic responses to acute stress and fluoxetine. Nat. Commun. 10, 350 (2019).

33. Sengupta, A., Bocchio, M., Bannerman, D. M., Sharp, T. \& Capogna, M. Control of Amygdala Circuits by 5-HT Neurons via 5-HT and Glutamate Cotransmission. J. Neurosci. 37, 1785-1796 (2017).

34. Robbins, T. W. \& Crockett, M. J. Role of Central Serotonin in Impulsivity and Compulsivity: Comparative Studies in Experimental Animals and Humans. Handbook of Behavioral Neuroscience 415427 (2010) doi:10.1016/s1569-7339(10)70093-x.

35. Blier, P., Piñeyro, G., el Mansari, M., Bergeron, R. \& de Montigny, C. Role of somatodendritic 5-HT autoreceptors in modulating 5-HT neurotransmission. Ann. N. Y. Acad. Sci. 861, 204-216 (1998).

36. Sharp, T. Serotonergic Feedback Control. Handbook of Behavioral Neuroscience 233-247 (2010) doi:10.1016/s1569-7339(10)70081-3.

37. Mendez-David, I. et al. Rapid Anxiolytic Effects of a 5-HT4 Receptor Agonist Are Mediated by a Neurogenesis-Independent Mechanism. Neuropsychopharmacology vol. 39 1366-1378 (2014).

38. Dulawa, S. C., Holick, K. A., Gundersen, B. \& Hen, R. Effects of chronic fluoxetine in animal models of anxiety and depression. Neuropsychopharmacology 29, 1321-1330 (2004).

39. Gartside, S. E., Umbers, V., Hajós, M. \& Sharp, T. Interaction between a selective 5-HT1A receptor antagonist and an SSRI in vivo: effects on 5-HT cell firing and extracellular 5-HT. British Journal of Pharmacology vol. 115 1064-1070 (1995).

40. Gobert, A., Rivet, J.-M., Cistarelli, L. \& Millan, M. J. Potentiation of the Fluoxetine-Induced Increase in Dialysate Levels of Serotonin (5-HT) in the Frontal Cortex of Freely Moving Rats by Combined Blockade of 5-HT1A and 5-HT1B Receptors with WAY 100,635 and GR 127,935. Journal of Neurochemistry vol. 68 1159-1163 (2002).

41. Kobayashi, K., Ikeda, Y., Haneda, E. \& Suzuki, H. Chronic Fluoxetine Bidirectionally Modulates Potentiating Effects of Serotonin on the Hippocampal Mossy Fiber Synaptic Transmission. Journal of Neuroscience vol. 28 6272-6280 (2008).

42. Kobayashi, K. et al. Reversal of hippocampal neuronal maturation by serotonergic antidepressants. Proceedings of the National Academy of Sciences vol. 107 8434-8439 (2010).

43. Pascual-Brazo, J. et al. Modulation of neuroplasticity pathways and antidepressant-like behavioural responses following the short-term (3 and 7 days) administration of the 5-HT4 receptor agonist RS67333. The International Journal of Neuropsychopharmacology vol. 15 631-643 (2012). 
44. Lucas, G. et al. Selective serotonin reuptake inhibitors potentiate the rapid antidepressant-like effects of serotonin4 receptor agonists in the rat. PLoS One 5, e9253 (2010).

45. Barch, D. M. et al. Function in the human connectome: task-fMRI and individual differences in behavior. Neuroimage 80, 169-189 (2013).

46. Llera, A., Wolfers, T., Mulders, P. \& Beckmann, C. F. Inter-individual differences in human brain structure and morphology link to variation in demographics and behavior. Elife 8, (2019).

47. Winkler, A. M., Renaud, O., Smith, S. M. \& Nichols, T. E. Permutation inference for canonical correlation analysis. Neuroimage 220, 117065 (2020).

48. Giorgi, A. et al. Brain-wide Mapping of Endogenous Serotonergic Transmission via Chemogenetic fMRI. Cell Rep. 21, 910-918 (2017).

49. Popova, N. K. \& Naumenko, V. S. Polymorphism of Serotonin 5-HT Receptors as the Basis of the Multifunctionality of Serotonin. Neuroscience and Behavioral Physiology vol. 42 161-166 (2012).

50. Zhuang, X. Altered Emotional States in Knockout Mice Lacking 5-HT1A or 5-HT1B Receptors. Neuropsychopharmacology vol. 21 52S-60S (1999).

51. Schweighofer, N. et al. Low-serotonin levels increase delayed reward discounting in humans. J. Neurosci. 28, 4528-4532 (2008).

52. Ishii, K. et al. A polymorphism of serotonin 2A receptor (5-HT 2A R) influences delay discounting. Personality and Individual Differences vol. 121 193-199 (2018).

53. Fink, L. H. L. et al. Individual Differences in Impulsive Action Reflect Variation in the Cortical Serotonin 5-HT2A Receptor System. Neuropsychopharmacology 40, 1957-1968 (2015).

54. Dipasquale, O. et al. Receptor-Enriched Analysis of functional connectivity by targets (REACT): A novel, multimodal analytical approach informed by PET to study the pharmacodynamic response of the brain under MDMA. Neurolmage vol. 195 252-260 (2019).

55. Deco, G. et al. Whole-Brain Multimodal Neuroimaging Model Using Serotonin Receptor Maps Explains Non-linear Functional Effects of LSD. Curr. Biol. 28, 3065-3074.e6 (2018).

56. Dipasquale, O. et al. Unravelling the effects of methylphenidate on the dopaminergic and noradrenergic functional circuits. Neuropsychopharmacology 45, 1482-1489 (2020).

57. Dukart, J. et al. Cerebral blood flow predicts differential neurotransmitter activity. Sci. Rep. 8, 4074 (2018).

58. Czachura, J. F. \& Rasmussen, K. Effects of acute and chronic administration of fluoxetine on the activity of serotonergic neurons in the dorsal raphe nucleus of the rat. Naunyn. Schmiedebergs. Arch. 
Pharmacol. 362, 266-275 (2000).

59. Vidal, R., Valdizán, E. M., Mostany, R., Pazos, A. \& Castro, E. Long-term treatment with fluoxetine induces desensitization of 5-HT4receptor-dependent signalling and functionality in rat brain. Journal of Neurochemistry vol. 110 1120-1127 (2009).

60. Haahr, M. E. et al. Central 5-HT4 receptor binding as biomarker of serotonergic tonus in humans: a [11C]SB207145 PET study. Mol. Psychiatry 19, 427-432 (2014).

61. Reardon, P. K. et al. Normative brain size variation and brain shape diversity in humans. Science vol. $3601222-1227$ (2018).

62. Seidlitz, J. et al. Transcriptomic and cellular decoding of regional brain vulnerability to neurogenetic disorders. Nat. Commun. 11, 3358 (2020).

63. Ohmura, Y., Tanaka, K. F., Tsunematsu, T., Yamanaka, A. \& Yoshioka, M. Optogenetic activation of serotonergic neurons enhances anxiety-like behaviour in mice. Int. J. Neuropsychopharmacol. 17, 17771783 (2014).

64. Winkler, A. M., Ridgway, G. R., Douaud, G., Nichols, T. E. \& Smith, S. M. Faster permutation inference in brain imaging. Neuroimage 141, 502-516 (2016).

65. Salvan, P. et al. Causal evidence of network communication in whole-brain dynamics through a multiplexed neural code. doi:10.1101/2020.06.09.142695.

66. Winkler, A. M., Webster, M. A., Vidaurre, D., Nichols, T. E. \& Smith, S. M. Multi-level block permutation. Neuroimage 123, 253-268 (2015).

67. Smith, S. M. et al. Resting-state fMRI in the Human Connectome Project. Neurolmage vol. 80 144-168 (2013).

68. Smith, S. M. et al. Functional connectomics from resting-state fMRI. Trends in Cognitive Sciences vol. 17 666-682 (2013).

69. Glasser, M. F. et al. The minimal preprocessing pipelines for the Human Connectome Project. Neuroimage 80, 105-124 (2013).

70. Griffanti, L. et al. ICA-based artefact removal and accelerated fMRI acquisition for improved resting state network imaging. Neurolmage vol. 95 232-247 (2014).

71. Arnatkeviciute, A., Fulcher, B. D. \& Fornito, A. A practical guide to linking brain-wide gene expression and neuroimaging data. Neuroimage 189, 353-367 (2019).

72. Tzourio-Mazoyer, N. et al. Automated Anatomical Labeling of Activations in SPM Using a Macroscopic Anatomical Parcellation of the MNI MRI Single-Subject Brain. Neurolmage vol. 15 273-289 
(2002).

73. Tullo, S. et al. Warping an atlas derived from serial histology to 5 high-resolution MRIs. Scientific Data vol. 5 (2018).

74. Park, M. T. M. et al. Derivation of high-resolution MRI atlases of the human cerebellum at 3T and segmentation using multiple automatically generated templates. Neuroimage 95, 217-231 (2014).

75. Chakravarty, M. M., Bertrand, G., Hodge, C. P., Sadikot, A. F. \& Collins, D. L. The creation of a brain atlas for image guided neurosurgery using serial histological data. Neuroimage 30, 359-376 (2006).

76. Winterburn, J. L. et al. A novel in vivo atlas of human hippocampal subfields using high-resolution 3T magnetic resonance imaging. Neurolmage vol. 74 254-265 (2013).

77. Treadway, M. T. et al. Illness progression, recent stress, and morphometry of hippocampal subfields and medial prefrontal cortex in major depression. Biol. Psychiatry 77, 285-294 (2015).

78. Van Essen, D. C. et al. The Human Connectome Project: a data acquisition perspective. Neuroimage 62, 2222-2231 (2012).

79. Salvan, P. et al. Multimodal Imaging Brain Markers in Early Adolescence Are Linked with a Physically Active Lifestyle. J. Neurosci. 41, 1092-1104 (2021).

\section{Figures}


a

HTR genes
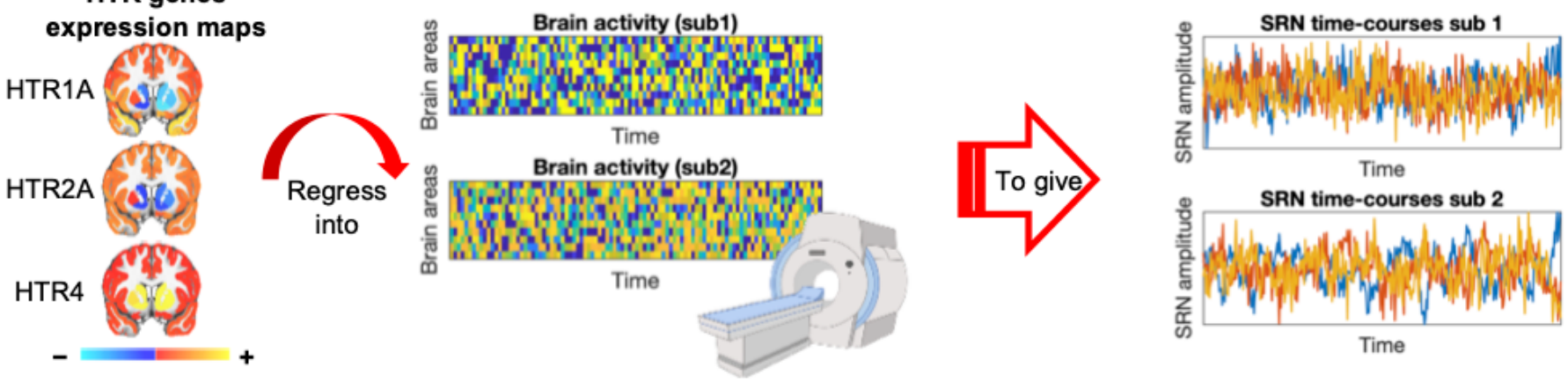

b

\section{Dual regression-stage 2}
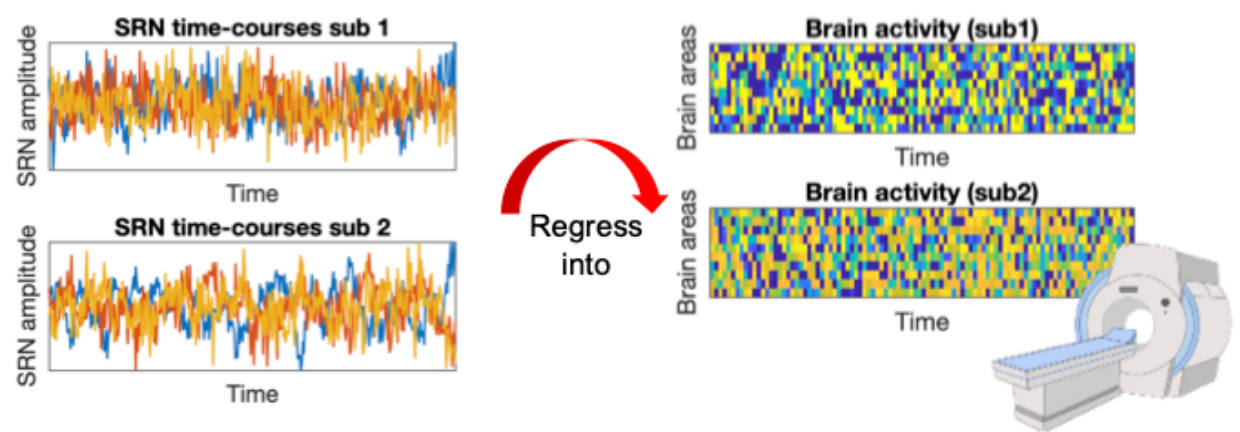

SRN FC maps
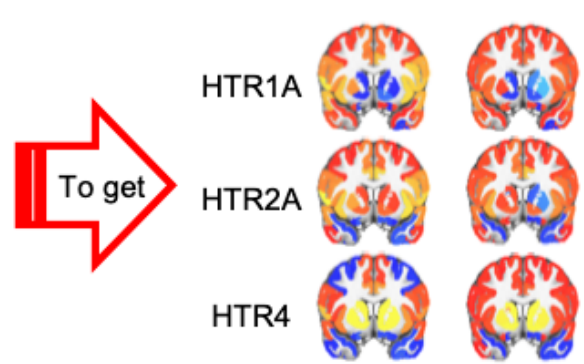

Sub 1

Sub 2

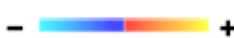

Figure 1

\section{Mapping functional signatures of brain-wide SRNs.}

Gene expression brain maps of serotonin receptor genes are combined with fMRI (both mouse and human) via FSL Dual Regression (DR) in order to map temporal and spatial fMRI signatures of different serotonin receptor types. a) DR-stage 1: regresses the spatial maps of serotonin receptor genes into each subject's 4D fMRI dataset. This gives a subject-specific time-course quantifying network amplitude changes for each SRN. DR-stage 1 can be used to address questions such as: What are the SRN amplitude changes in response to optogenetic stimulation? b) DR-stage 2: regresses subject-specific time-courses into the same 4D fMRI dataset. This provides a subject-specific spatial map quantifying functional connectivity (FC) for each SRN. DR-stage 2 can be used to address questions such as: What are the SRN brain correlates associated with individual differences in a depression scale? 


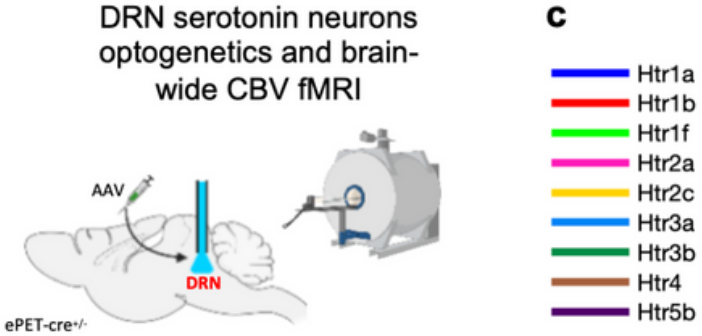

$\Rightarrow$ SRNs

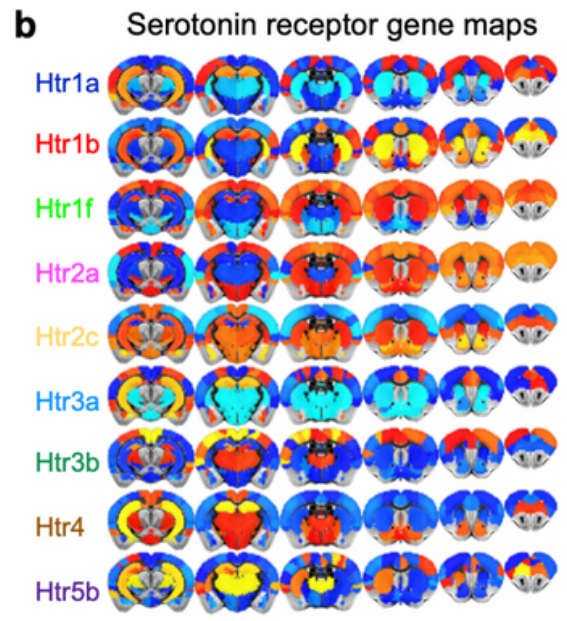

$-2=0=2$

Expression level (Z-scored) d

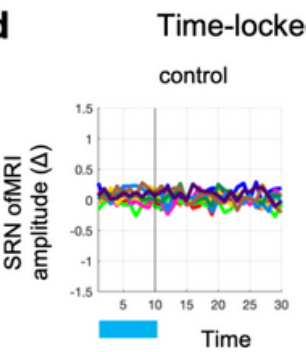

e

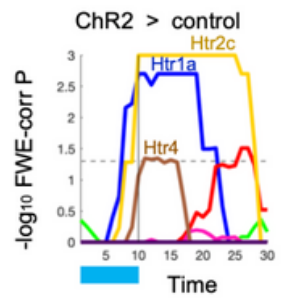

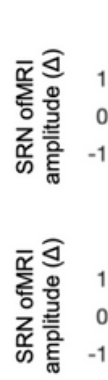

$-1-$
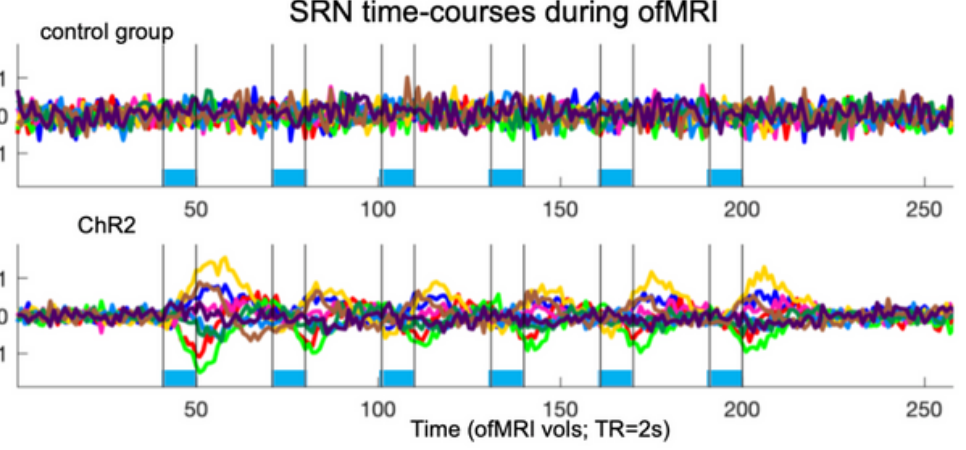

SRN time-courses during ofMRI

$\mathbf{f}$

SRN ofMRI functional connectivity

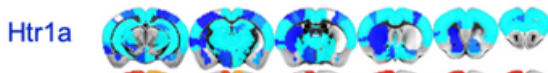

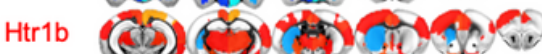

Htr1f $A$ or th th an?

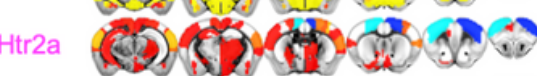

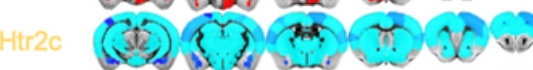

$\mathrm{Htr} 3 \mathrm{a}$

$\mathrm{Htr} 3 \mathrm{~b}$

Htr4

$\mathrm{Htr} 5 \mathrm{~b}$

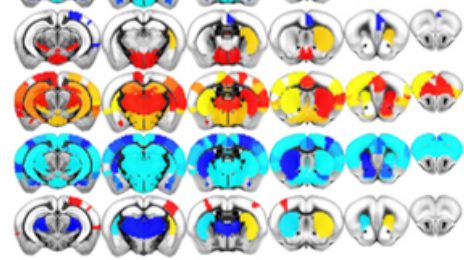

$\begin{gathered}\text { control > } \\ \text { ChR2 }\end{gathered} \quad 0=3 \begin{aligned} & \text { ChR2 > } \\ & \text { controls }\end{aligned}$ -log10 FWE-corr p-value

\section{Figure 2}

\section{Activation of DRN serotonin neurons modulates brain-wide SRNs.}

a) Mice were genetically manipulated to express ChR2 in DRN serotonin neurons prior to ofMRI experiments ${ }^{32}$. b) Transcriptomic maps of serotonin receptor genes from the Allen Brain Institute. The combination of serotonin receptor maps and ofMRI via FSL dual regression allowed to characterise SRNs. c) SRN time-courses of amplitude changes (DR-stage 1 ) during ofMRI. ( $\Delta=$ changes from baseline activity, see Fig. S2a). Top row shows SRN amplitude changes for control animals; bottom row for ChR2 animals. Each color represents a SRN; SRN colors are matched with the other panels. d) Time-locked ofMRI amplitude changes of SRNs in control animals (left) and in ChR2 animals (right). Blue bar underneath represents when optogenetic stimulation was on. e) Results from permutation-based inference testing on SRN time-locked responses between control and ChR2 animals. Showing -log10 FWE-corr p-values, corrected across time, SRNs, and two tails. Dotted lines demarcate statistical thresholds. Together, panels $\mathbf{d}$ ) and $\mathbf{e}$ ) show a heterogeneity of SRN amplitude changes in response to optogenetic activation of DRN serotonin neurons. f) Results from permutation-based inference testing on SRN functional connectivity maps (DR-stage 2) between control and ChR2 groups. Showing -log10 FWEcorr P, corrected across brain regions, SRNs, and two tails. Cold colors represent decreased functional connectivity in the ChR2 group compared to the control group; hot colors represent increased functional connectivity. 

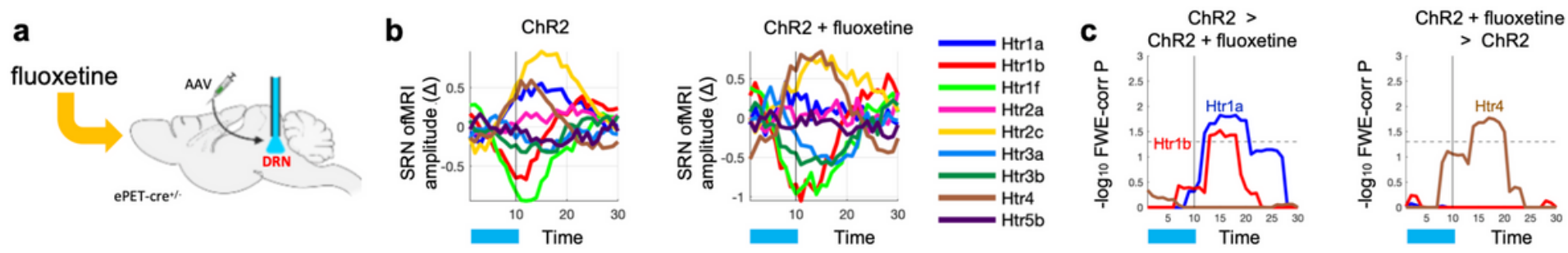

Figure 3

Fluoxetine manipulation alters neuromodulation in expected SRNs.

a) ChR2 animals were treated with one pharmacologically significant dose of fluoxetine and then underwent ofMRI. b) Time-locked ofMRI amplitude changes of SRNs in ChR2 animals (left) and in ChR2 animals treated with fluoxetine (right). Each color represents a SRN. c) Results from permutation-based inference testing for group differences in $\mathrm{Htr} 1 \mathrm{a}, \mathrm{Htr} 1 \mathrm{~b}, \mathrm{Htr} 4 \mathrm{SRN}$ time-locked amplitude changes, between the ChR2 group and the ChR2 group treated with fluoxetine. Showing -log10 FWE-corr p-values, corrected across time and two tails. Dotted lines demarcate statistical thresholds. 

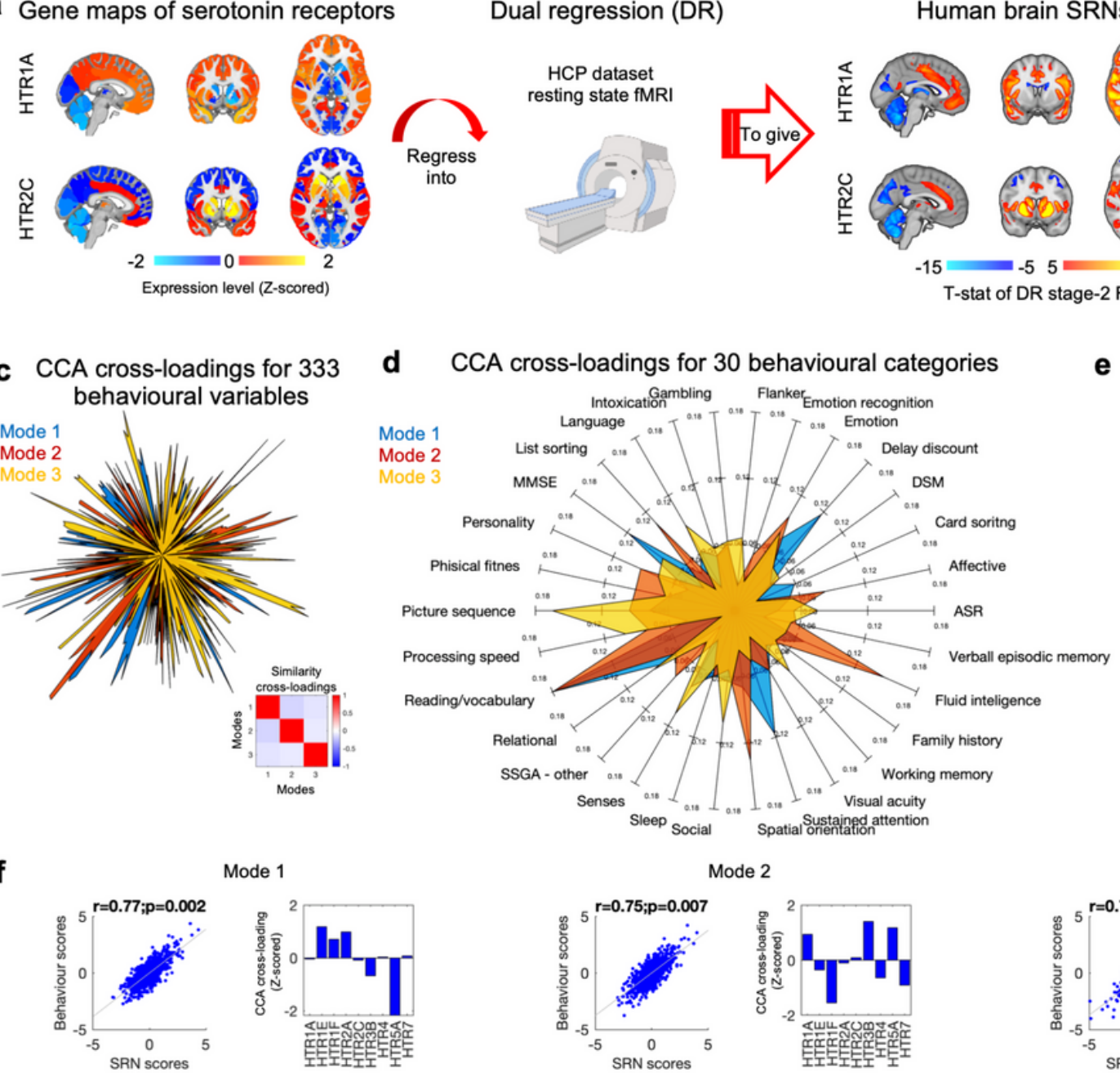

eCA cross-loadings for delay

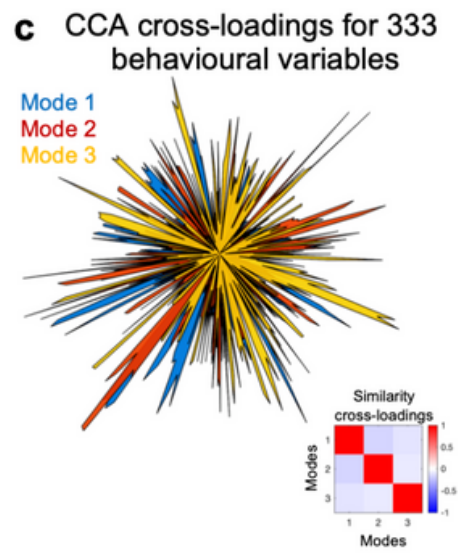

Mode 2

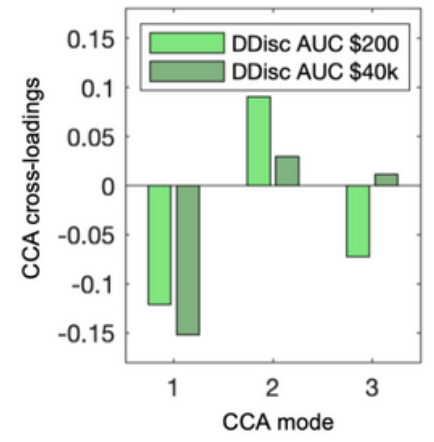

f

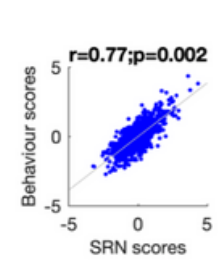

Mode 1
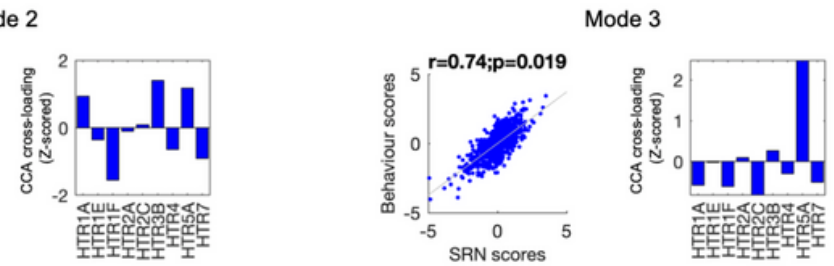

g
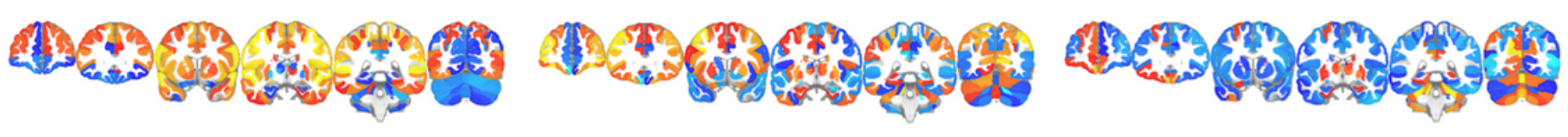

Figure 4

Brain-wide SRNs are linked with human behaviour via latent modes of population variation.

a) FSL DR approach to derive SRNs in the HCP dataset. b) individual differences in SRNs were tested for covariation with differences in behaviours via canonical correlation analysis (CCA). Permutation inference CCA characterised three strong, statistically significant modes of population variation. These modes are orthogonal and link specific sets of brain-wide SRNs (imaging cross-loadings) with specific phenotypes of human behaviour (behavioural cross-loadings). c) Top: Showing radial plot of (absolute) CCA cross-loadings for all 333 behavioural variables, depicting three behavioural phenotypes. Bottom: Similarity between cross-loadings (or behavioural phenotypes). d) CCA cross-loadings depicted in panel c) were summarised into 30 behavioural categories in order to ease interpretation of behavioural phenotypes. In both $\mathbf{c}$ ) and d), CCA modes 1, 2, and 3, are color-coded respectively in blue, red, yellow. e) Cross-loadings for delay discounting (DDisc) variables. (AUC = area under the curve). $\mathrm{f}$ ) For each CCA 
mode, showing (left) scatter plot of SRN and behaviour covariates (each dot is a subject), and (right) CCA SRN cross-loadings (averaged across brain regions, one bar per SRN). g) For each CCA mode, showing brain maps of imaging cross-loadings (averaged across SRNs). As the CCA was carried out on ROI-based SRN functional connectivity values, maps are displayed preserving this level of resolution. For the complete set of CCA imaging cross-loadings (across all SRNs and across all brain regions) see Fig. S4.

\section{Supplementary Files}

This is a list of supplementary files associated with this preprint. Click to download.

- floatimage2.png

- floatimage4.png

- floatimage6.png

- floatimage8.png 